\title{
A Pole-Zero Based Criterion for Optimal Placement of Collocated Sensor-Actuator Pair
}

\author{
Dimitri Piron ${ }^{\mathrm{a}, *}$, Shashank Pathak ${ }^{\mathrm{a}}$, Arnaud Deraemaeker ${ }^{\mathrm{b}}$, Christophe Collette $^{\mathrm{a}, \mathrm{c}}$ \\ ${ }^{a}$ BEAMS Department, Université Libre de Bruxelles, Brussels, Belgium \\ ${ }^{b}$ BATir Department, Université Libre de Bruxelles, Brussels, Belgium \\ ${ }^{c}$ Department of Aerospace and Mechanical Engineering, Université de Liège, Liège, Belgium
}

\begin{abstract}
A new and computationally efficient criterion is developed concerning the optimal placement of a collocated sensor-actuator pair for active control of structural vibrations with a low authority controller. The basic idea behind the proposed criterion is based on the maximization of the pole-zero distance in open-loop which has a direct link with the maximum achievable damping ratio once in closed-loop. Unlike poles, the determination of transmission zeros is computationally tedious because it depends upon the placement of the sensor-actuator pair. Therefore, a new approximation to reliably estimate the transmission zeros is also introduced which remarkably enhances the computational efficiency of the proposed optimization criterion. The effectiveness of the proposed criterion is demonstrated on a cantilever beam and a simply supported plate and is also compared with two other widely used criteria: the Gramian controllability and the spatial $H_{2}$ norm. It is shown that the proposed criterion is pertinent and ensures higher damping values for the cantilever beam compared to the other two criteria. Nonetheless, the criterion requires adaptations to improve its reliability for structure with large modal density such as a simply supported plate.
\end{abstract} Keywords: optimal placement, optimization criterion, sensors-actuators, transmission zero, pole-zero distance, vibration control

\section{Introduction}

Nowadays, for technical, economical and environmental reasons, large and lightweight structures are widely used in advanced applications where high performance is required. Unfortunately, such large structures are also increasingly sensitive to vibrations, preventing them from meeting specified requirements and introducing negative consequences at different degrees of severity: operational errors (such as the attitude stability degradation in satellites [1, 2]), structural damage (e.g. induced by fatigue [3, 4]) or loss of comfort for the end user (for instance due to the spurious noise in the automotive industry [5, 6]). Hence, to ensure the intended performance of the structure despite its weak vibration robustness, a vibration control strategy has to be implemented for reducing the vibration level while ensuring high performance and stability.

\footnotetext{
* Corresponding author

Email address: dimitri.piron@ulb.be (Dimitri Piron)
} 
During the control architecture conception, although a proper design of the control law that successfully meets robustness and performance is critical for the vibration alleviation, the appropriate number of the transducers to use as well as their suitable positioning on the system to be controlled passively or actively is also a well established concern. Gupta et al. [7] thoroughly reviewed the studies related to the selection of best positions of piezoelectric transducers on smart structures and highlighted the fact that the major difficulty related to optimal sensors and actuators $(\mathrm{SA})$ placement is that the obtained positioning solution highly depends on the criterion used to perform the optimization, impacting therefore the performance of the controlled structure. Hence, the optimization criterion needs to be chosen with utmost care.

For example, Maghami et al. [8] and Williams [9] optimized the SA placement for flexible space structures by maximizing the real part of the open-loop transmission zeros. This optimization criterion relies on the mathematical property that those open-loop zeros characterize the asymptotic behaviour of the closed-loop poles submitted to high control gain. Hence, maximizing the real part of those zeros ensures small settling time so fast regulation. Hac and Liu [10] introduced the use of the controllability and observability Gramians as performance indexes for the SA placement, which has been widely used in various problems such as for the optimization of the location of piezoelectric SA on a simply supported beam [11, for the positioning of piezoelectric patches on plates [12, 13] or for the piezoelectric positioning on a large flexible structure [14]. This approach is based on the maximization of the energy from the inputs to the states (actuator placement) and from the states to the outputs (sensor placement), again in the open-loop system.

Another concept based on the open-loop system for the optimization of the SA placement is to use the $\mathrm{H}_{2}$ norm to find the position that maximises the importance of the pair over the structure in the sense of the amplitude of vibration the input can induce at the output [15]. The $H_{2}$ norm criterion has also been extended by Moheimani and Ryall to the spatial $H_{2}$ norm [16], allowing to compute the average input-output characteristics over a domain of interest. Several SA placement optimizations have been conducted using the spatial $H_{2}$ norm, such as piezoelectric patch placement on a thin plate [17] or the reduction of the spillover phenomena [18].

All those criteria are rather simple to implement and do not require large computational efforts but present a major drawback. Indeed, these criteria do not account for the additional dynamics brought by the controller in the system as they only focus on open-loop optimization. Considering that a strong interaction between this extra dynamics and the one from the plant is highly probable, performing the SA optimization in open-loop can lead to sub-optimal solutions, as demonstrated for instance by Devasia et al. [19]. To overcome this notable inconvenience, SA optimization can be performed in closed-loop configuration by adding the control law to the optimization process. For example, Kang et al. [20] optimized, for a laminated beam, the piezoelectric SA placement and gain values with a state feedback controller by maximizing modal damping. Recently, even more advanced controllers have been used such as the Linear-Quadratic Regulator (LQR) for the optimization of piezoelectric patches location on plates [21, 22, 23].

Although including the control law in the optimization process certainly delivers improved 
performance compared to the ones obtained with open-loop optimizations [19], the computational costs drastically increase because the optimization has to be performed together for the SA positions and for the controller parameters.

Considering this trade-off between performance and complexity, this paper proposes a novel and efficient open-loop criterion for achieving the optimal positioning of a collocated SA pair for closed-loop systems controlled by a low authority controller (LAC). The proposed optimization criterion is based on the maximization of the open-loop pole-zero distance, which has a direct link with the maximum achievable damping ratio in closed-loop. It allows therefore to benefit from the computational efficiency aspect of an open-loop criterion while ensuring high performance in closed-loop.

The first section of this paper presents the concept of the proposed pole-zero distance criterion for the placement of collocated SA pairs. The second part details how the performance criterion based on this approach is built. The third section compares the effectiveness of the proposed criterion with other techniques on simple structures. Finally, the concluding remarks are given.

\section{The Pole-Zero Distance Concept}

The transmission zeros (also called anti-resonances) of a single-input and single-output (SISO) linear and time-invariant system are defined as the frequencies at which a non-zero actuation leads to a zero output in the response. Hence, when considering the transfer function of the system from the actuator to the sensor, the transmission zeros $z$ correspond to the frequencies at which the transfer function equals to zero. Unlike the poles that are global properties of the structure and its boundary conditions, the value of those transmission zeros depends on the position of the SA in the system.

The transmission zeros play an important role in the dynamical behaviour of the system and are therefore essential for control theory. For instance, the poles of the closed-loop system tend to the open-loop zeros for high value of the feedback gain. Moreover, a zero provides a phase lead which balances the phase lag brought by the pole and allows therefore to obtain a better phase margin. Furthermore it has been proven by Martin [24] that collocated systems present alternating poles and zeros (interlacing property) which ensures that the open-loop system phase lag can never be more than 180 degrees. For such collocated system, the distance between the pole and the zero to which it converges when the gain is increased, for a specific mode, has a direct influence on the maximum achievable damping ratio once a feedback control law is added to the system. For example, when a lead controller is applied and when the zero value $z_{i}$ of the $i$ th mode is not larger than three times the value of the natural frequency $\omega_{i}$ of the same mode, the maximal reachable damping ratio of this mode $\xi_{i}^{\max }$ can be expressed by [25]:

$$
\xi_{i}^{\max } \approx \frac{z_{i}-\omega_{i}}{2 z_{i}}
$$

It is clear from this result that the relative value of the transmission zero with respect to the pole has a direct link with the maximal reachable damping ratio. Because the positioning of the SA 
on the system directly influences the transmission zero values, the proposed optimization criterion for the placement of a collocated SA pair on a system is based on the maximization of the pole-zero distance $|P Z|_{i}$, as illustrated in Fig. 1. It can be seen that changing the position of a SA pair on the structure $\left(\mathbf{x}_{\mathbf{1}}, \mathbf{x}_{\mathbf{2}}\right.$ and $\left.\mathbf{x}_{\mathbf{3}}\right)$ does not modify the pole value $\omega$ but affects the transmission zero $z$. Thus, moving the SA pair on the structure changes the relative pole-zero distance (in this specific case defined by $\left.z_{i}-\omega\right)$ and therefore the SA pair can be placed on the structure in an optimum way to maximize the maximum achievable damping ratio $\xi^{\text {max }}$.

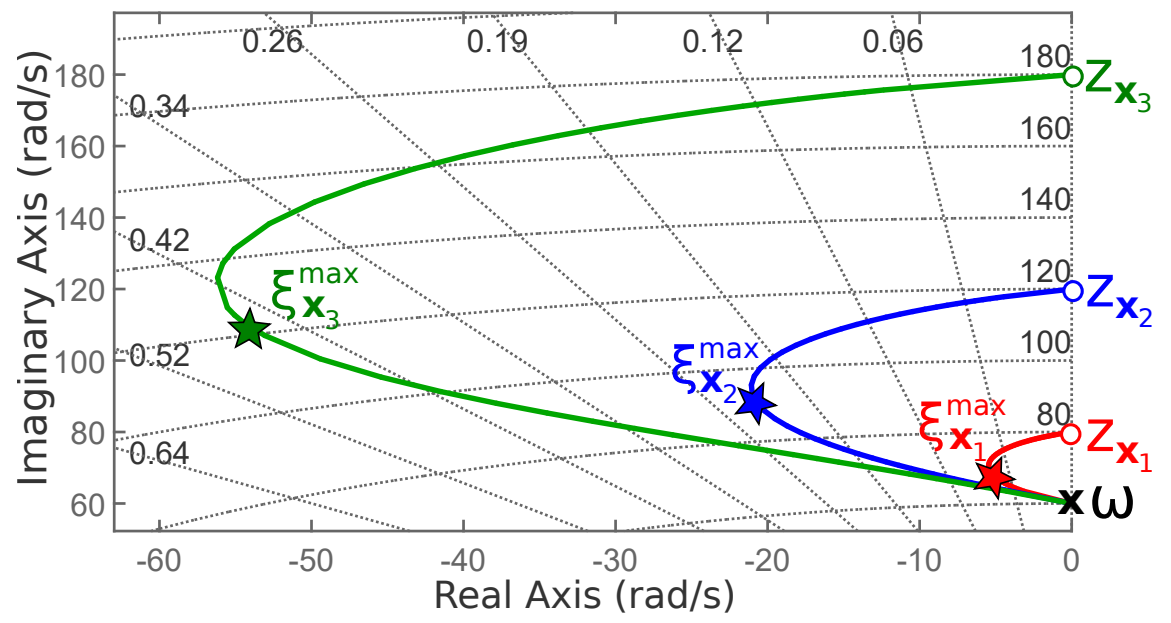

Figure 1: Graphical Illustration of the Influence of the Relative Pole-Zero Distance on the Maximum Reachable Damping Ratio for Three SA Positions

Establishing the SA positioning optimization based on the pole-zero distance criterion allows therefore, due to the meaning of this pole-zero distance, to perform the optimization in open-loop system (limiting consequently the computational cost) while ensuring high modal damping ratio once the LAC is applied.

\subsection{Computation of the Transmission Zero}

Considering that the proposed criterion is based on the pole-zero distance maximization, the zero values $z$ must be accurately known for all the SA pair positioning candidates on the structure. The most straightforward method consists of solving the equation obtained by setting the transfer function to zero:

$$
G(\omega)=0
$$

When a continuous structure is modelled with a numerical approximation, it leads to a discretized system with $n$ degrees of freedom (DOF) for which the dynamical behaviour can be represented by the equations of motion in the matrix form:

$$
M \ddot{p}+C \dot{p}+K p=D u
$$


where $p \in \mathbb{R}^{n}$ is the physical displacements vector, $u \in \mathbb{R}^{r}$ is the control vector with $r$ being the number of actuators, $D \in \mathbb{R}^{n \times r}$ is the applied force distribution matrix and $M, C$ and $K \in \mathbb{R}^{n \times n}$ are, respectively, the mass, damping and stiffness matrices. The natural frequencies $\omega_{i}(i=1,2$, $\ldots, n)$ and each corresponding mode shape $\phi_{i} \in \mathbb{R}^{n}$ of such system can be computed by solving the eigenvalue problem obtained when setting $C$ and $D$ of Eq. 3 to zero (i.e. when considering the free response of the undamped system). It is then possible to perform a change of coordinates from the physical to the modal ones according to:

$$
p=\Phi q
$$

where $\Phi \in \mathbb{R}^{n \times n}$ is the modal matrix defined by $\Phi=\left(\phi_{1}, \phi_{2}, \ldots, \phi_{n}\right)$ and $q \in \mathbb{R}^{n}$ is the vector of modal coordinates. Performing this mapping between the physical space to the modal one allows to obtained decoupled equations and to express the transfer function in its matrix form as a sum of each modal contribution [24]:

$$
G(\omega)=\sum_{i=1}^{n} \frac{\phi_{i} \phi_{i}^{T}}{\mu_{i}\left(\omega_{i}^{2}+2 j \xi_{i} \omega_{i} \omega-\omega^{2}\right)}
$$

where $\mu_{i}$ and $\xi_{i}$ are respectively the modal mass and the modal damping ratio associated to the $i^{\text {th }}$ mode. Therefore, the transfer function between a collocated SA pair located at a specific position $\mathbf{x}=\alpha$ can be expressed by:

$$
G(\omega)_{\alpha}=\sum_{i=1}^{n} \frac{\phi_{i}^{2}(\alpha)}{\mu_{i}\left(\omega_{i}^{2}+2 j \xi_{i} \omega_{i} \omega-\omega^{2}\right)}
$$

Substituting Eq. 6 into Eq. 2 shows that obtaining the transmission zeros for a specific SA configuration requires to solve, for $\omega$, a $2 \times(n-1)^{t h}$ order equation. Hence, for realistic structures that generally consist of a large number of DOFs, it would be computationally too expensive to build the transfer function and then to solve the related problem for each candidate in order to obtain the pole-zero distance.

Another method to obtain the transmission zeros values utilizes the physical meaning of those zeros. Indeed, it has been proved that for displacement or velocity sensors, the transmission zeros of a specific architecture correspond to the natural frequencies of the same structural system which is constrained at the SA location, in the direction of the SA pair [26]. It is therefore possible to obtain the transmission zero values by solving the eigenvalue problem of the constrained system, e.g. [27]. However, in case of realistic structures with a high number of DOFs, solving the eigenvalue problem for each SA position considered would be again too expensive in terms of computational cost.

The transmission zeros can also be obtained using the state space formulation where they are expressed by the frequencies at which the rank of a specific subspace (built with the state, input and output matrices) is reduced. Although this method could be more efficient with respect to the other two, it still presents three major drawbacks: (1) the state space formulation has to be built for each SA location, (2) the computational cost depends on the number of DOFs in the system and 
(3) it can be numerically difficult to obtain the solutions, requiring thereby extra computational method [28, 29, 30].

A common issue with these methods is that their computational complexity increases significantly with the number of DOFs of the system. Moreover, the zero corresponding to a defined SA position can be computed only after building a specific system (the transfer function, the constrained equivalent system or the state space), consequently preventing the determination of the zero values for multiple SA positions at the same time. In view of the above, a computationally efficient approximation method is proposed in the next section to estimate the transmission zeros with reasonable accuracy. It can be used for a specific mode only and allows to simultaneously obtain the zero values for all the SA positioning candidates.

\subsection{Transmission Zero Approximation}

Because the frequency bandwidth of interest is always limited, a modal truncation can be performed such that only the first $m$ modes (with $m<n$ ) are retained. Therefore, the transfer function of a collocated SA pair at the specific position $\mathbf{x}=\alpha$ can be expressed in its matrix form by Eq. 7

$$
\begin{gathered}
G(\omega)_{\alpha}=\sum_{i=1}^{m} \frac{\phi_{i}^{2}(\alpha)}{\mu_{i}\left(\omega_{i}^{2}+2 j \xi_{i} \omega_{i} \omega-\omega^{2}\right)}+R_{\alpha} \quad(m<n) \\
R_{\alpha}=\left(K^{-1}\right)_{\alpha, \alpha}-\sum_{i=1}^{m} \frac{\phi_{i}^{2}(\alpha)}{\mu_{i} \omega_{i}^{2}}
\end{gathered}
$$

where $R_{\alpha}$ is the static correction of high order modes at $\alpha$ that does not depend on the frequency and ensures high accuracy of the system dynamics despite the truncation [31]. By assuming that the mode shapes have been normalised to obtain unitary modal masses and that the structure presents only small damping allowing to neglect the imaginary part of the pole, the transfer function (Eq. 7) can be rewritten in the Laplace domain as:

$$
G(s)_{\alpha}=\frac{\phi_{1}^{2}(\alpha)}{s^{2}+\omega_{1}^{2}}+\ldots+\frac{\phi_{i-1}^{2}(\alpha)}{s^{2}+\omega_{i-1}^{2}}+\frac{\phi_{i}^{2}(\alpha)}{s^{2}+\omega_{i}^{2}}+\frac{\phi_{i+1}^{2}(\alpha)}{s^{2}+\omega_{i+1}^{2}}+R_{\alpha}
$$

where $s$ is the complex Laplace variable. Let us search for an estimation of the transmission zero that lies between $\omega_{i}$ and $\omega_{i+1}$. The modal contribution of mode $(i+1)$ is not simplified by its static contribution because the dynamic behaviour of this mode in the vicinity of the resonance $\omega_{i+1}$ can have evident influence on the targeted zero value. By approximating the modal contributions of modes 1 to $(i-1)$ by their roll-off component as described in Eq. 10, Eq. 9 is simplified into Eq.

$$
\begin{gathered}
\frac{1}{s^{2}+\omega_{j}^{2}} \simeq \frac{1}{s^{2}} \quad(j=1,2, \ldots, i-1) \\
G(s)_{\alpha}=\frac{\sum_{k=1}^{i-1} \phi_{k}^{2}(\alpha)}{s^{2}}+\frac{\phi_{i}^{2}(\alpha)}{s^{2}+\omega_{i}^{2}}+\frac{\phi_{i+1}^{2}(\alpha)}{s^{2}+\omega_{i+1}^{2}}+R_{\alpha}
\end{gathered}
$$


Since the transmission zeros can be obtained by equating the transfer function to zero, the following $6^{\text {th }}$ order equation is obtained, which is satisfied by the zeros of the system:

$$
a s^{6}+b s^{4}+c s^{2}+d=0
$$

where the four parameters $a, b, c$ and $d$ depend on the mode shapes of modes 1 to $(i+1)$ and the natural frequencies $\omega_{i}$ and $\omega_{i+1}$ only. It may be noted that Eq. 12 can be analytically solved as a cubic equation in $s^{2}$, which provides six solutions for $s$. Due to the use of the residual term for high frequency modes and the roll-off component for the low frequency ones, the approximation is only valid between $\omega_{i}$ and $\omega_{i+1}$, which is precisely the frequency bandwidth in which the zero of mode $i$ is located because of the alternating pole-zero property. The solution of Eq. 12 should therefore be chosen carefully such that it satisfies the following inequality:

$$
\omega_{i}^{2}<z_{i}^{2}<\omega_{i+1}^{2}
$$

Hence, the proposed approximation allows to easily compute the transmission zeros corresponding to a specific mode for all the desired SA positions at the same time, for a lightly damped system. This is feasible considering that it only requires simple arithmetic calculations. The accuracy of the approximation is discussed in the next section with a numerical simulation.

\subsection{Numerical Validation of the Transmission Zero Approximation}

The numerical simulation has been performed using the Structural Dynamics Toolbox (SDT), an open and extendible finite element modelling MATLAB based toolbox for dynamics problems 32. For illustration, two different systems have been considered: a cantilever beam and a simply supported plate. Both system are defined with the same lightly damped steel with the following properties: Young's modulus $E=210 \mathrm{GPa}$, Poisson's ratio $\nu=0.285$, mass density $\rho=7800$ $\mathrm{kg} / \mathrm{m}^{3}$ and modal damping ratio $\xi=0.004$. The cantilever beam is modelled with the dimension of $300 \mathrm{~mm}$ x $25 \mathrm{~mm}$ x $2 \mathrm{~mm}$ and its finite element model consists of 601 beam elements where the 2 degrees of freedom allowing the out-of-plane bending motion are assigned to each node of the model, except for the first one at the fixed end of the cantilever beam. Consequently, the model consists of 1200 degrees of freedom in total. As illustrated in Fig. 2a, each non-constrained node along the longitudinal axis of the beam is defined as a potential collocated force actuator/displacement sensor pair position, leading to 600 possible locations. The simply supported plate is modelled with the dimension of $310 \mathrm{~mm}$ x $250 \mathrm{~mm}$ x $2 \mathrm{~mm}$ and is discretized by 6 DOFs shell elements each $5 \mathrm{~mm}$ in the $x$ and $y$ directions (3213 nodes in total). The nodes located at the four edges of the plate allow only the three rotations, leading to 18606 DOFs in total. A SA pair is allowed each $10 \mathrm{~mm}$ in both directions (i.e. one out of two nodes), leading to $775 \mathrm{SA}$ positioning candidates. The system representation as well as its Cartesian coordinates are shown in Fig. 2b

To assess the computational efficiency of the proposed approximation, the value of the zero that stands between the $3^{\text {rd }}$ and the $4^{\text {th }}$ mode of the defined cantilever beam is computed for all the possible SA positions using three methods: (1) solving the eigenvalue problem of the 


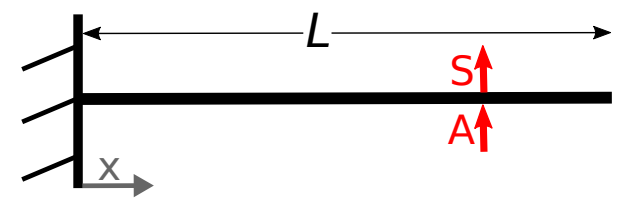

(a)

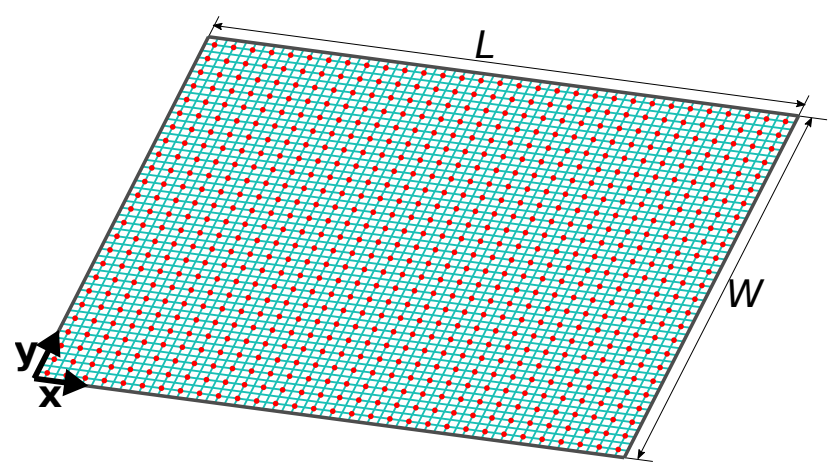

(b)

Figure 2: Representation of the Two Structural Systems: (a) The Cantilever Beam with a SA Position Candidate Along its Longitudinal Axis $x$, (b) The Simply Supported Plate with all the SA Positioning Candidates in Red Dots

corresponding constrained system, (2) equating the transfer function to zero and (3) using the proposed approximation method. Because the transmission zero physically corresponds to the natural frequency of the corresponding constrained system, the values obtained with this method can be defined as references for all the SA positions. Hence, the relative error of a zero value can be defined as the ratio between the absolute error and its equivalent reference where the absolute error corresponds to the difference between the obtained zero value and its reference. Table 1 shows, for each approach, the maximum relative error value obtained among all the SA positions as well as the related computational time. Note that all computations have been performed on a 64-bit Windows 10 platform computer with an Intel(R) Core(TM) i5-8400 at 2.8GHz and 16Gb of RAM. It can be seen that although each method provides the same order of magnitude for the relative error, the proposed approximation computes the 600 transmission zeros up to 500 times faster than the reference technique. This therefore demonstrates that the proposed approximation is highly efficient in terms of computational cost.

The maximum relative error value obtained among all the possible SA positions for the first six modes are shown in Fig. 3 It can be seen for the cantilever beam (Fig. 3a) that the error is limited to only a few percent while it goes up to $16 \%$ for the simply supported plate (Fig. 3b). This disparity can be explained by the difference in the modal density between the two systems. The cantilever beam presents indeed modes that are well separated, which complies with the rolloff approximation (Eq. 10) and the simplification of the higher order modes dynamics into their static contributions. There is however a strong modal interaction between nearby modes for the plate, which decreases the accuracy of the approximation and leads to the observed maximum error values.

The proposed zero approximation is therefore highly efficient in terms of computational cost and provides accurate values for systems with small modal density. Nevertheless, it can lead to bigger deviations once applied to systems with high modal density. An improvement of its current form could be performed in a future study in order to reduce the deviation for such systems. 
Table 1: Maximum Relative Error and Processing Time When Computing the Zero That Lies Between the 3rd and the $4 t h$ Mode for 600 SA Positions with Three Different Methods

\begin{tabular}{l|c|c}
\hline \hline \multicolumn{1}{c|}{ Method } & $\begin{array}{c}\text { Maximum } \\
\text { Relative Error (\%) }\end{array}$ & $\begin{array}{c}\text { Processing } \\
\text { Time (s) }\end{array}$ \\
\hline (1) Solving the constrained system & 0 & 184.75 \\
(2) Equating $G(\omega)$ to zero & 1 & 885.12 \\
(3) Proposed Approximation & 1.1 & 0.36 \\
\hline \hline
\end{tabular}

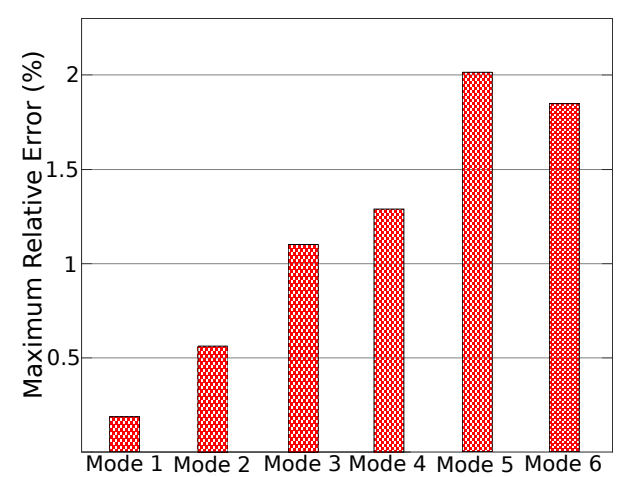

(a) Beam

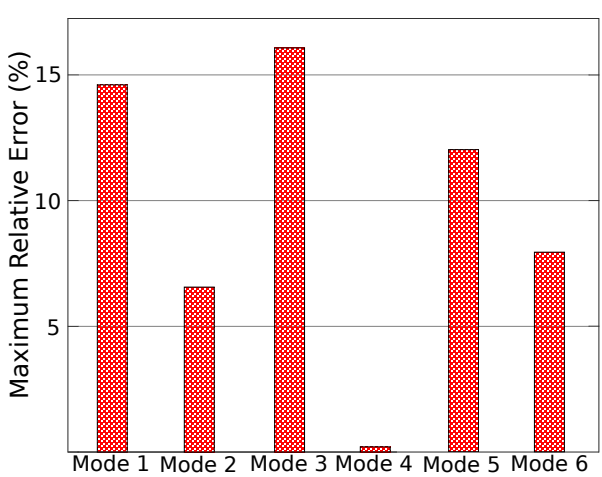

(b) Plate

Figure 3: Maximum Relative Error of the Proposed Zero Approximation for the First Six Modes: (a) Results for the Cantilever Beam, (b) Results for the Simply Supported Plate

\section{Proposed Pole-Zero Distance Criterion}

To achieve the maximum damping ratio as shown in Fig. 1 the pole-zero distance needs to be maximized. It is worth mentioning here that the pole-zero distance refers to the distance between the pole of the mode to be controlled and the zero it tends to when the feedback gain becomes infinitely large.

When considering a LAC with a collocated SA pair, the root locus usually exhibits a number of disconnected loops similar to Fig. 11. The pole-zero distance of the $i^{\text {th }}$ mode $|P Z(\mathbf{x})|_{i}$ can therefore always be computed using the same procedure but requires to know in advance which type of control law will be used. For example, mechanical systems with lead controller present, in closed-loop, a pole before each zero [25]. The pole-zero distance is consequently obtained by the difference between the zero and the pole of the targeted mode $i$, as follow:

$$
|P Z(\mathbf{x})|_{i}=z_{i}(\mathbf{x})-\omega_{i}
$$

where $\mathbf{x}$ is the SA position on the structure and $z_{i}(\mathbf{x})$, the zero of the targeted mode, can be computed using the approximation developed in Section 2

To ensure high performance in terms of modal damping, the proposed criterion consists of three factors: (i) the first one focuses on overall maximization of pole-zero distances. Therefore, it is represented as an arithmetic mean of the normalized pole-zero distances corresponding to all the modes to be damped. It is worth mentioning here that the pole-zero distances are normalized 
with respect to the distance between two neighbouring poles around the zero, i.e. the maximum achievable pole-zero distance. This normalization ensures that each modal distance has the same weight in the criterion even if it is related to a higher-order mode. Subsequently, (ii) the second factor is required to ensure that each individual mode of interest presents a maximum possible pole-zero distance. Therefore, this second factor is represented by the geometric mean of all the normalized pole-zero distances, dropping the value of the criterion to zero for SA positions where pole-zero cancellation happens for any of the modes of interest. Finally, (iii) a third factor is also required to avoid the condition of pole-zero cancellation caused when the adjacent zero of the mode of interest lies too close to the pole of the targeted mode. Such situation is illustrated in Fig. 4 for a lead controller where it can be seen that position $\mathbf{x}_{1}$ (Fig. 4a) presents two controllable modes while position $\mathbf{x}_{2}$ (Fig. 4b) leads to a loss of controllability of the second mode due to the first zero that lies too close to $\omega_{2}$. Hence, the third term of the criterion is assigned to the geometric mean of the normalized pole-zero distances computed with the pole of the mode of interest and its adjacent zero.

Consequently, the proposed SA placement criterion for a collocated system with LAC that ensures high modal damping ratios for the targeted modes is defined as follow:

$$
J(\mathbf{x})=\left(\frac{1}{n} \sum_{k} \frac{|P Z(\mathbf{x})|_{k}}{|P Z(\mathbf{x})|_{k, \text { max }}}\right) \times\left(\sqrt[n]{\prod_{k} \frac{|P Z(\mathbf{x})|_{k}}{|P Z(\mathbf{x})|_{k, \text { max }}}}\right) \times\left(\sqrt[n]{\prod_{k} \frac{\left|P_{k} Z_{a d j}(\mathbf{x})\right|}{\left|P_{k} Z_{a d j}(\mathbf{x})\right|_{\text {max }}}}\right)
$$

where $k$ contains the $n$ modes of interest, $|P Z(\mathbf{x})|_{k}$ is the pole-zero distance of the targeted mode at position $\mathbf{x}$ and $\left|P_{k} Z_{a d j}(\mathbf{x})\right|$ corresponds to the adjacent pole-zero distance. Following this, the SA position that maximises the index function introduced by Eq. 15 will ensure the best compromise between the different maximum achievable modal damping ratios for the $n$ targeted modes once the feedback control law is added to the system.

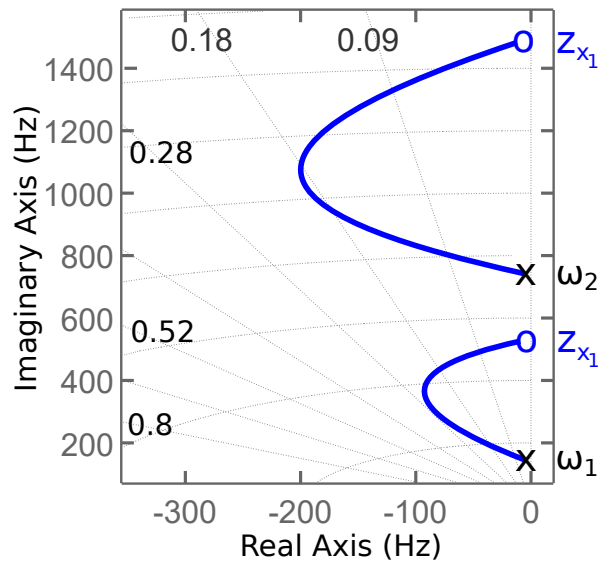

(a)

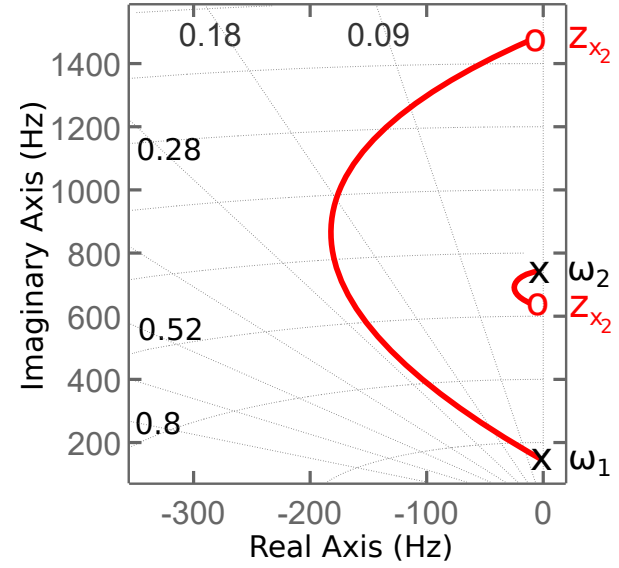

(b)

Figure 4: Graphical Illustration of the Loss of Controllability due to Adjacent Zero when a Lead Control is Applied: (a) modes 1 and 2 are controllable, (b) only mode 1 is controllable 


\section{Numerical Examples}

In this section, the proposed pole-zero distance criterion is applied for determining the placement of a collocated force actuator/displacement sensor pair that maximizes the modal damping ratio for targeted modes of interest. The two systems used in Section 2 and illustrated by Fig. 2 are used again, i.e. the cantilever beam (600 SA positioning candidates along its longitudinal axis) and the simply supported plate (775 SA positioning candidates on its surface). For both systems, it is chosen to use a lead controller once the loop will be closed. The criterion given by Eq. 15 can therefore be rewritten as:

$$
J(\mathbf{x})=\left(\frac{1}{n} \sum_{k} \frac{z_{k}(\mathbf{x})-\omega_{k}}{\omega_{k+1}-\omega_{k}}\right) \times\left(\sqrt[n]{\prod_{k} \frac{z_{k}(\mathbf{x})-\omega_{k}}{\omega_{k+1}-\omega_{k}}}\right) \times\left(\sqrt[n]{\prod_{k} \frac{\omega_{k}-z_{k-1}(\mathbf{x})}{\omega_{k}-\omega_{k-1}}}\right)
$$

where the pole-zero distance is computed by the difference between the zero and the pole of the targeted mode. The third term is computed by the difference between the pole linked to the mode of interest and the previous zero. Different cases are considered: single mode damping (mode 1 , mode 2 and mode 3 ) and multi-modal damping (modes $1 \& 2$ and modes $2 \& 3$ ). For each position and for each targeted mode(s), the criterion is computed using Eq. 16 and the zero approximation provided in Section 2

Figure 5 shows $\tilde{J}(\mathbf{x})$ for all the possible SA positions along the beam for single mode damping where $\tilde{J}(\mathbf{x})$ corresponds to the normalization of $J(\mathbf{x})$ by its maximum obtained value and $L$ is the length of the beam. For the damping of the first mode, the criterion has its maximum value at a distance of $0.7833 \mathrm{~L}$ away from the clamped end of the beam. For the second and third modes, the positions that maximize the pole-zero criterion are respectively at a distance of $0.49 \mathrm{~L}$ and $0.3367 \mathrm{~L}$ from the clamped end. Moreover and as expected, the criterion value drops to zero in the vicinity of the modal nodes. For multi-modal damping, the positions that maximize the criterion are at a distance of $0.9166 L$ for modes $1 \& 2$ and $0.3566 L$ for modes $2 \& 3$, as shown in Fig. [6 The results obtained for the simply supported plate are available in Table 2 and illustrated for the multi-modal damping case of modes $1 \& 2$ in Fig. 7 where $L$ is the length of the plate and $W$ is its width. As expected, the criterion value drops to zero in the vicinity of the four edges of the simply supported plate, but also in the vicinity of the nodal line of the second mode, proving again the benefit of the third factor in the criterion. Of course, the performance obtained with those different positions has to be analysed in closed-loop, which is implemented in the next section.

\subsection{Closed-loop Performance}

The closed-loop damping achieved by the optimal positions obtained with the proposed polezero distance criterion are analysed and compared with the ones obtained using two other open-loop criteria: the Gramian controllability and the spatial $H_{2}$ norm.

By injecting the change of coordinates from the physical to the modal ones according to Eq. 4 into the equations of motion of the system (Eq. 3 ) and by (1) considering that the modal matrix $\Phi$ 


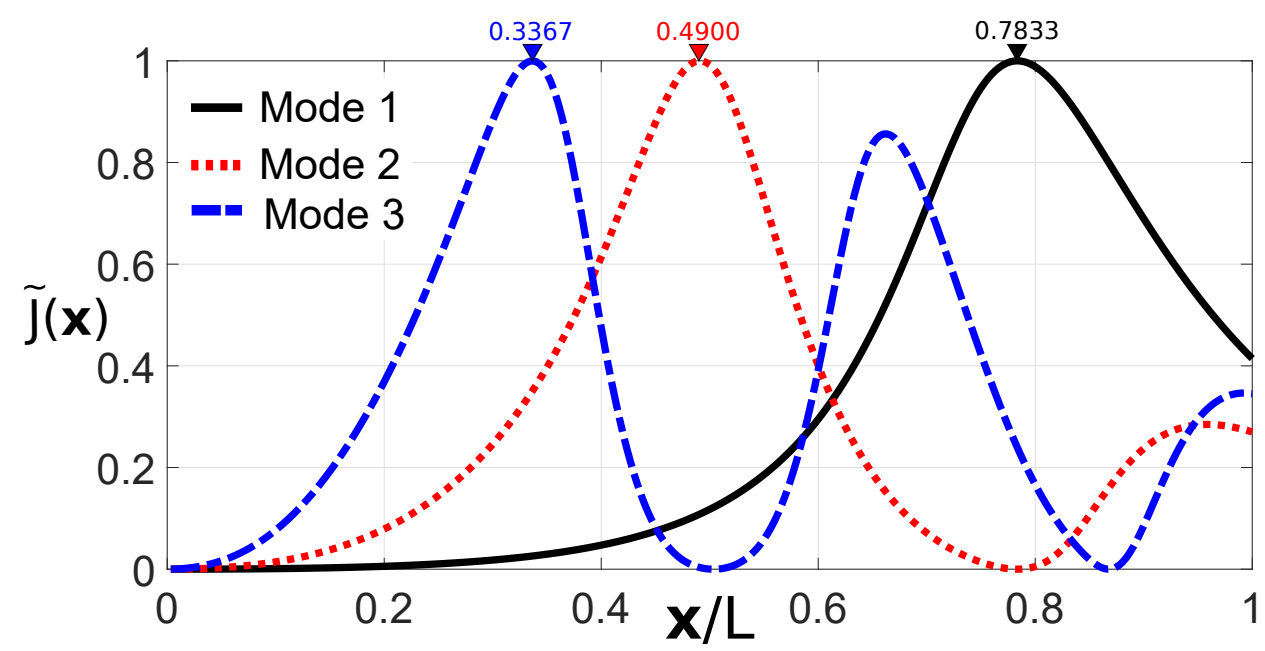

Figure 5: Normalized Criterion Values on the Longitudinal Axis $(x / L)$ of the Beam for Modes 1,2 and 3

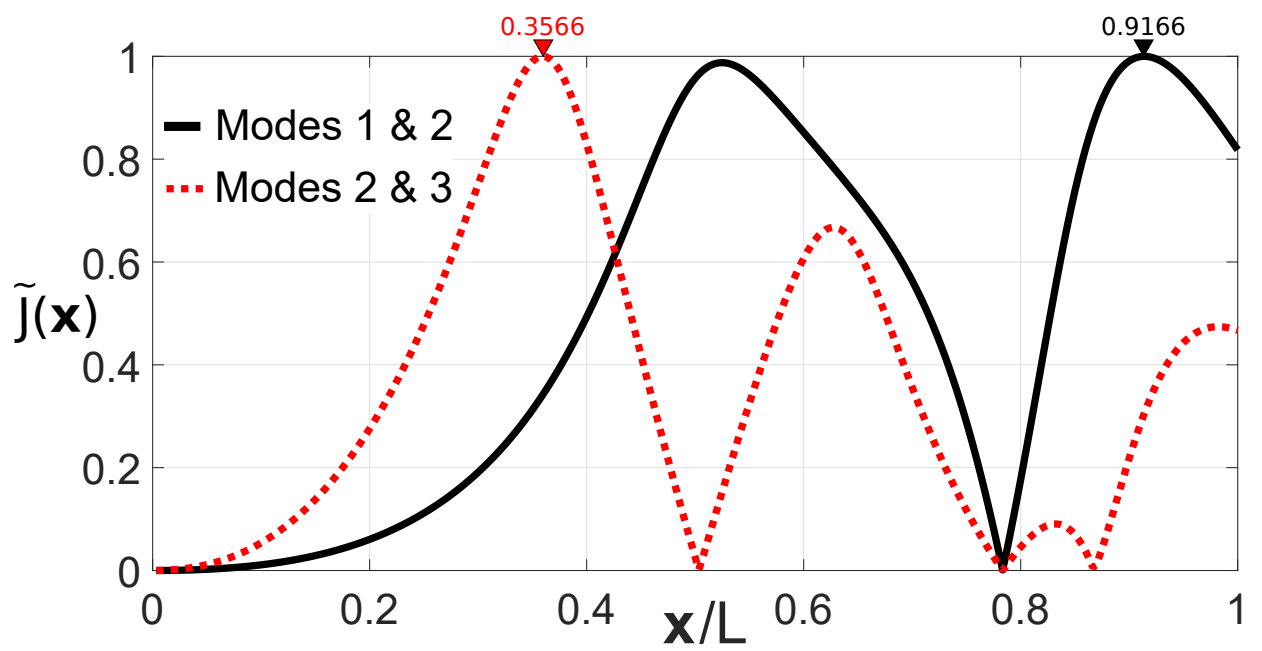

Figure 6: Normalized Criterion Values on the Longitudinal Axis $(x / L)$ of the Beam for Modes $1 \& 2$ and Modes 2 $\& 3$

Table 2: Normalized Positions $(x / L, y / W)$ on the Simply Supported Plate that maximize $J(\mathbf{x})$ for the Different Modal Cases

\begin{tabular}{c|c|c|c|c}
\hline \hline Mode 1 & Mode 2 & Mode 3 & Modes 1 \& 2 & Modes 2 \& 3 \\
\hline \multirow{3}{*}{$(0.5,0.5)$} & & & & $(0.339,0.38)$ \\
& $(0.113,0.5)$ & $(0.5,0.3)$ & $(0.371,0.5)$ & $(0.661,0.38)$ \\
& $(0.887,0.5)$ & $(0.5,0.7)$ & $(0.629,0.5)$ & $(0.339,0.62)$ \\
& & & & $(0.661,0.62)$ \\
\hline \hline
\end{tabular}




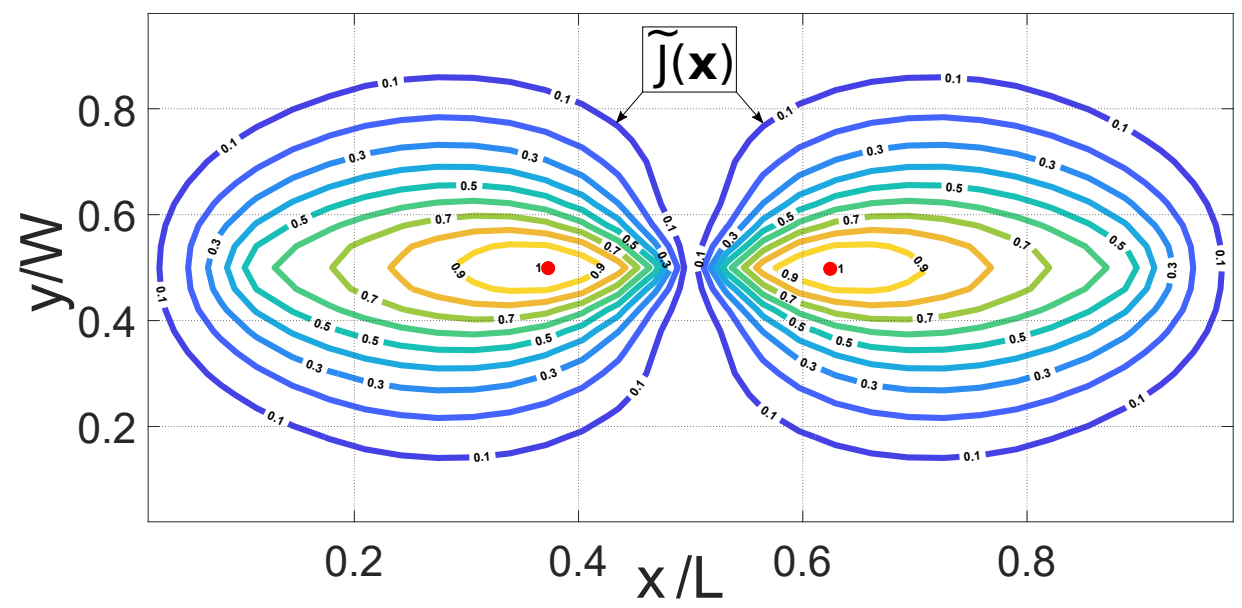

Figure 7: Normalized Criterion Values Contour on the Simply Supported Plate Considering the Simultaneous Optimization of Modes 1 \& 2

has been normalised with respect to the mass matrix $M$ and (2) defining the state variable vector $x$ by $x=[q ; \dot{q}] \in \mathbb{R}^{2 n}$, the modal state-space formulation of the system is given by:

$$
\begin{gathered}
\dot{x}=A x+B u \\
\text { with } A=\left[\begin{array}{cc}
0 & I \\
-\Omega^{2} & -2 \xi \Omega
\end{array}\right], \quad B=\left[\begin{array}{c}
0 \\
\Phi^{T} D
\end{array}\right]
\end{gathered}
$$

where $\Omega=\operatorname{diag}\left(\omega_{i}\right)$ and $\xi=\operatorname{diag}\left(\xi_{i}\right)$. If the system is subjected to an external disturbance which forces it towards the initial state $x_{0}$, the aim of the control architecture is to bring it back to the desired state $x_{t}$. Therefore, by defining the signal energy as the integral of the square of the signal magnitude [33], it is possible to find the minimum control signal energy $u$ required to bring back the system from $x_{0}$ to $x_{t}$, in a certain amount of time $t$. The solution of this linear quadratic problem provides the optimal control input signal which leads to the total control energy expressed by $[10]$ :

$$
\int_{0}^{t}\|u(\tau)\|^{2} d \tau=\left(e^{A t} x_{0}-x_{t}\right)^{T} W_{c}(t)^{-1}\left(e^{A t} x_{0}-x_{t}\right)
$$

where $W_{c}(t)$ is the controllability Gramian matrix defined by:

$$
W_{c}(t)=\int_{0}^{t} e^{A \tau} B B^{T} e^{A^{T} \tau} d \tau
$$

The Gramian controllability criterion is based on the selection of the proper position for the actuators that minimizes the total control energy, i.e. the position that maximizes the eigenvalues of $W_{c}$. If $\lambda_{k}$ is defined as the eigenvalue of $W_{c}$ linked to the specified mode of interest $k$, the higher $\lambda_{k}$ is, the easier mode $k$ can be controlled and, subsequently, the less control energy it requires to attenuate the structural vibrations of this mode. The optimal SA position obtained with the controllability Gramian criterion is therefore the one that maximises the Gramian controllability index $J_{\text {Gram }}(\mathbf{x})$ defined by [13]: 


$$
J_{\text {Gram }}(\mathbf{x})=\left(\sum_{k} \lambda_{k}\right) \times\left(\sqrt{\prod_{k} \lambda_{k}}\right)
$$

Because the actuators position only impacts the input matrix $B$, this maximization of $J_{\text {Gram }}(\mathbf{x})$ is directly related to the modal amplitude of the targeted mode $\phi_{k}$. It has been indeed mathematically demonstrated by Hac and Liu [10] that for a lightly damped structure with well spaced natural frequencies, the $\mathrm{k}^{t h}$ eigenvalue of $W_{c}$ when the actuator is located at a specific position $\mathbf{x}=\alpha$ is expressed by:

$$
\lambda_{k}(\alpha)=\frac{\phi_{k}(\alpha)^{2}}{4 \xi_{i} \omega_{i}}
$$

The $\mathrm{H}_{2}$ norm of a SISO linear and time-invariant system described by its transfer function $G(\omega)$ is defined by [15]:

$$
\|G\|_{2}=\sqrt{\frac{1}{2 \pi} \int_{-\infty}^{\infty} \operatorname{tr}\left(G^{*}(\omega) G(\omega)\right) d \omega}
$$

and can be interpreted as the average gain of the system subjected to an impulse response, which therefore provides the response characteristics of the system at the sensor location. The spatial $H_{2}$ norm $\ll G \gg_{2}$ has been introduced to measure the response of the system over a defined subset $\mathcal{R}$ and is mathematically defined by [16]:

$$
\ll G \gg_{2}=\sqrt{\frac{1}{2 \pi} \int_{-\infty}^{\infty} \int_{\mathcal{R}} \operatorname{tr}\left(G^{*}(\omega, r) G(\omega, r)\right) d r d \omega}
$$

It may be noted that injecting the expression of the transfer function (Eq. 5] into Eq. 23 provides a demonstration of the direct link between $\ll G \gg_{2}$ and the modal amplitudes $\phi_{i}$, as already observed for the Gramian controllability. When the SA positioning optimization is performed with the spatial $H_{2}$ criterion, the optimal location is the one that maximises the spatial $H_{2}$ controllability index $J_{H_{2}}(\mathbf{x})[16]$ :

$$
\begin{aligned}
J_{H_{2}}(\mathbf{x})= & \frac{1}{\beta} \sqrt{\sum_{k} f_{k}(\mathbf{x})} * 100 \\
& \text { with } f_{k}(\mathbf{x}) / \alpha_{k} \geqslant b_{k}
\end{aligned}
$$

where $f_{k}(\mathbf{x})$ is the spatial $H_{2}$ norm of mode $k, \beta$ is the maximum value of the criterion obtained among all the candidates, $\alpha_{k}$ is the maximum value of $f_{k}(\mathbf{x})$ among all the candidates and $b_{k}$ is a threshold value for the controllability of mode $k$. The spatial $H_{2}$ norm $f_{k}(\mathbf{x})$ is a measure of the actuator authority on mode $k$ over the defined subset $\mathcal{R}$. Hence, maximizing $J_{H_{2}}(\mathbf{x})$ while ensuring high enough value for each $f_{k}(\mathbf{x})$ through $b_{k}$ allows to maximize the average authority on all the targeted modes without a loss of modal authority. By fixing the threshold value $b_{k}$ to 0.5 for all the targeted modes as done in [16] and by setting $\mathcal{R}$ as all the SA position candidates, the optimal positions on the two studied systems for the different modes of interest are computed using Eqs. 20 and 24. For the cantilever beam, the optimal positions for both criteria and regardless 
of the modal case are always located at its free end, which actually corresponds to the position where the mode shape values for the different targeted modes are maximum. The results for the simply supported plate are available in Fig. 8 in which the optimal positions obtained with the proposed criterion are included as well. It can be observed for single mode control (Fig. 8a that the controllability Gramian as well as the spatial $H_{2}$ norm criteria provide once again optimal SA locations where the value of the mode shapes are maximum, which confirms the observations made regarding the link between the two criteria and the modal amplitudes.

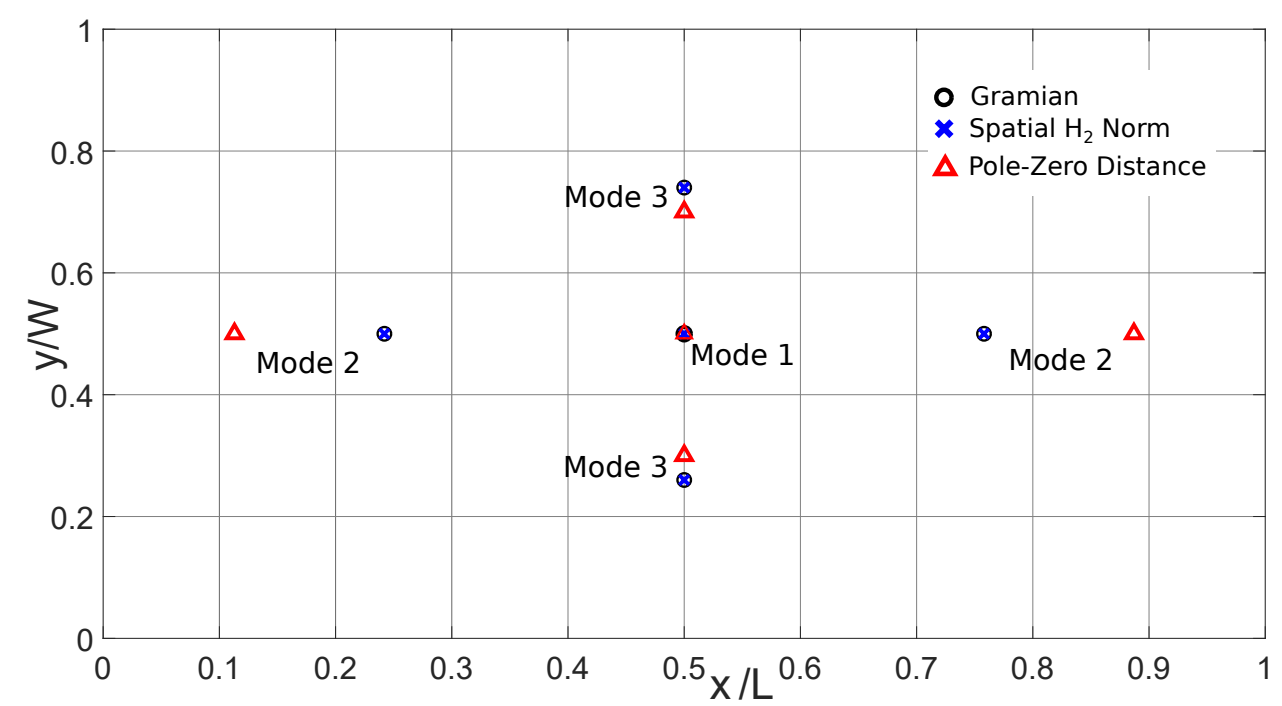

(a)

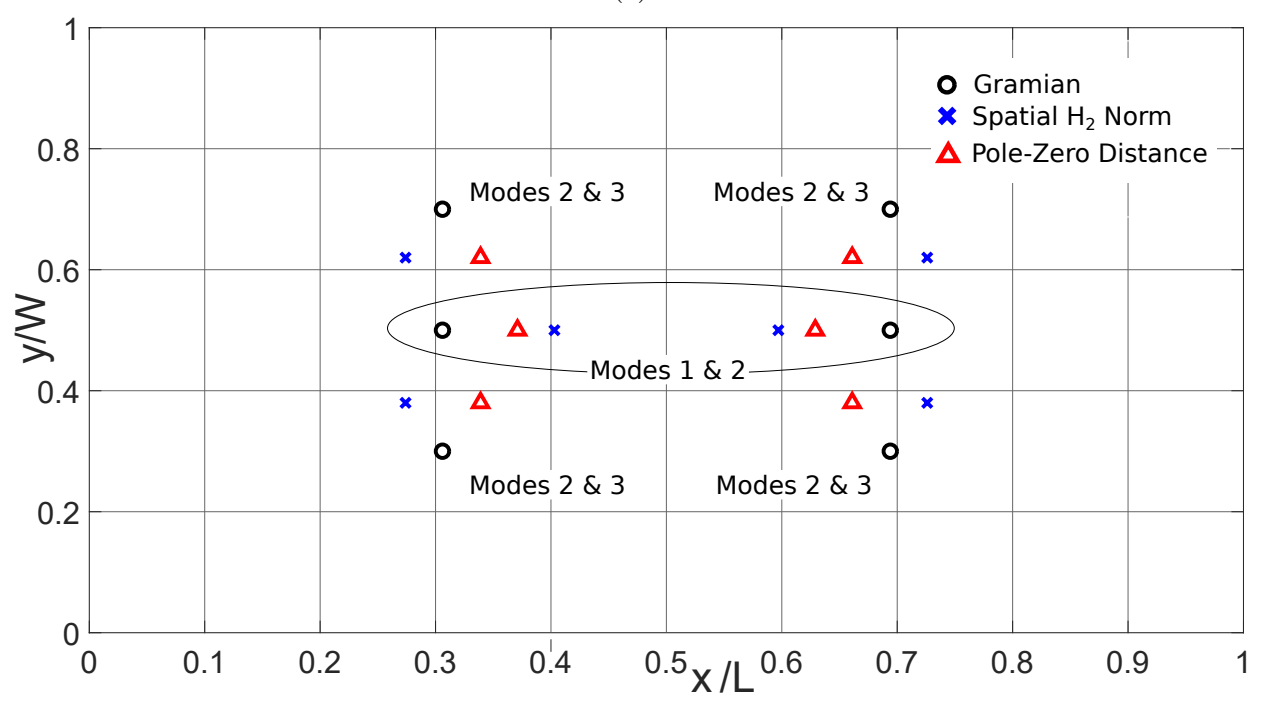

(b)

Figure 8: Optimal Positions Obtained on the Simply Supported Plate with the Three Criteria: (a) Single Optimization for Modes 1, 2 and 3, (b) Simultaneous Optimization for Modes $1 \& 2$ and Modes $2 \& 3$

To analyse the closed-loop performance provided by those different positions, a lead control as described by Eq. 25 is considered [34]. In order to have a comprehensive comparison, the zero $z_{c}$ and the pole $p_{c}$ of the controller only depend on the targeted modes as follow: $z_{c}=\frac{\omega_{p}}{2}$ and $p_{c}=2 * \omega_{q}$ where $\omega_{p}$ and $\omega_{q}$ are respectively the smallest and highest eigen frequencies of 
the targeted modes. The value of the gain $g$ is tuned for each position in order to maximize the damping ratios using the cost function provided by Eq. 26. where $k$ contains all the considered modes.

$$
\begin{gathered}
C(s)=g \frac{s+z_{c}}{s+p_{c}} \\
J_{\text {Gain }}(g)=\left(\sum_{k} \xi_{k}\right) \times\left(\sqrt{\prod_{k} \xi_{k}}\right)
\end{gathered}
$$

The modal damping ratios obtained for single mode cases and the different criteria are compared in Fig. 9, For the cantilever beam (Fig. 9a), it is clear that the proposed criterion provides higher damping ratios compared to the Gramian or the spatial $H_{2}$ norm criteria. It can be explained by the fact that those two criteria only depend on the mode shape of the targeted mode. Conversely, the proposed pole-zero distance criterion is derived using the contribution of the surrounding modes when computing the zero value, including therefore modal interaction even if the optimization is performed in the open-loop system. Hence, the information is more rich, which leads to better performance in terms of damping.

Regarding the results for the simply supported plate (Fig. 9b), the damping obtained for mode 1 and mode 3 are within the same range for the different criteria. However, mode 2 shows less than a half of the damping value when the proposed approach is used. This can be explained by the high modal density for simply supported plate and the cancellation of the third and fourth modes in the vicinity of the obtained position, which leads to a local reshaping of the root locus and, therefore, a sub-optimal prediction of the pole-zero distance despite the use of a LAC.

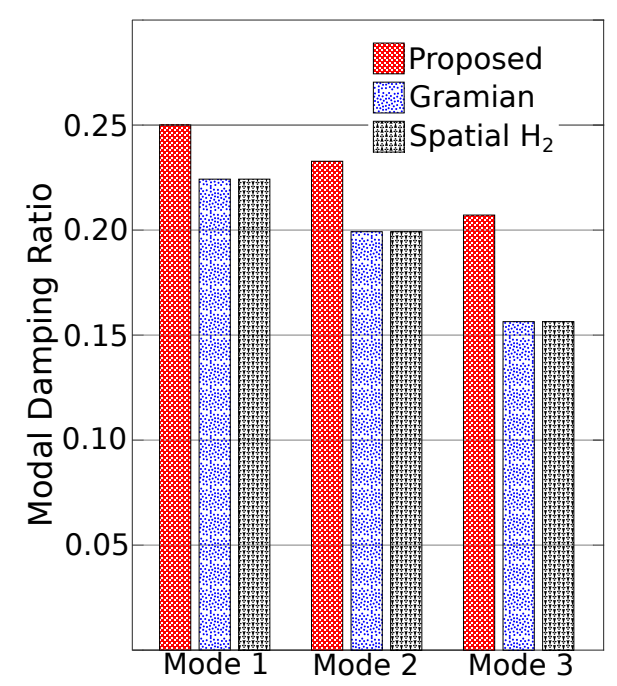

(a) Beam

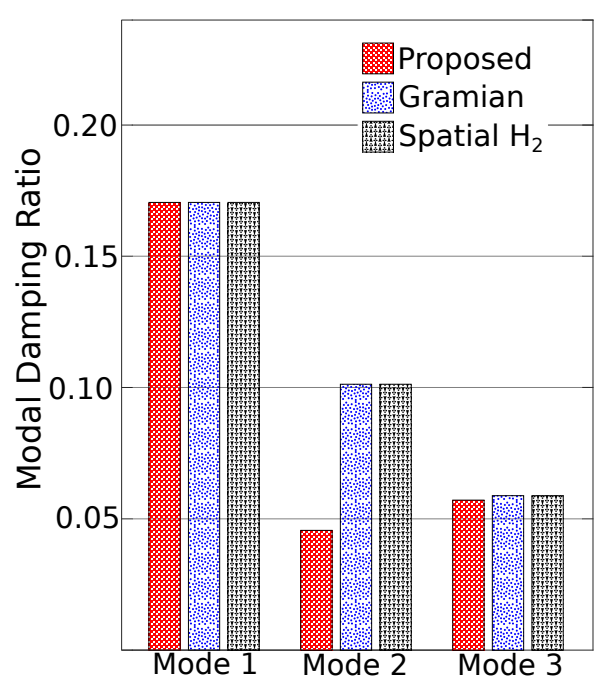

(b) Plate

Figure 9: Damping Ratios in Closed-Loop for Single Mode Optimization: (a) Results for the Cantilever Beam, (b) Results for the Simply Supported Plate

The comparison of the damping obtained with the different criteria for multi-modal cases is 
available in Fig. 10. It can be observed for all the cases and for both systems that the proposed pole-zero criterion allows to have a better compromise between the different modes to damp. Indeed, the other criteria provide a bigger difference between the two damping ratios, which can result in sub-performance due to modal interaction: if one of the two modes to control shows a high modal damping value while the other one is significantly less damped, the dynamical amplification of this less damped mode can reduce the overall damping performance in the frequency band of interest. The effectiveness of the proposed criterion can be verified with the frequency response functions obtained in case of multi-modal damping optimization of, for example, modes $1 \& 2$ on the cantilever beam and on the simply supported plate. To ensure the excitation of the two modes, the force disturbance is located at the free end of the beam and at the position $(0.31 L, 0.50 \mathrm{~W})$ of the plate. In both cases, the performance measurement is achieved by a displacement sensor which is collocated with the disturbance and the control architectures (i.e. the position of the transducers and the parameters of the control law) are implemented with the previously obtained results. The different frequency response functions can be found in Fig. 11 where it can be observed that the proposed criterion shows better performance for the cantilever beam and, as suggested, a better compromise for the simply supported plate, which confirms therefore the effectiveness of the proposed criterion.

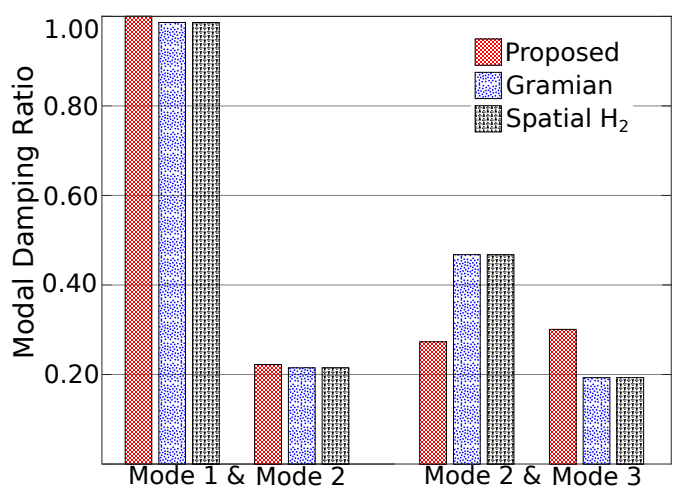

(a) Beam

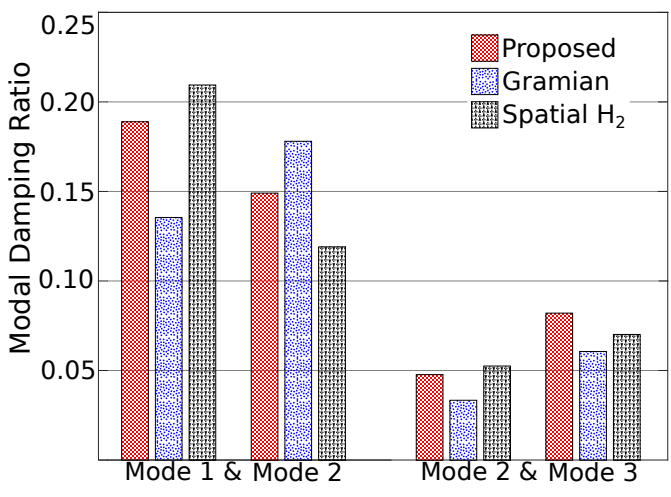

(b) Plate

Figure 10: Damping Ratios in Closed-Loop for Multi-modal Optimization: (a) Results for the Cantilever Beam, (b) Results for the Simply Supported Plate

\section{Conclusions and Perspectives}

This paper presents a new and efficient criterion based on the maximization of the pole-zero distance to find the optimal location for a collocated SA control pair. The approach is based on the direct link between the pole-zero distance and the maximum achievable damping ratio of the mode, allowing to perform the optimization in open-loop.

To perform fast estimation of the zero values, a new computational scheme based on the approximation of the transfer function is also introduced. This approximation allows to compute the transmission zeros corresponding to a specific mode for all the SA positioning candidates at the same time, which leads to a large decrease of the processing time. 


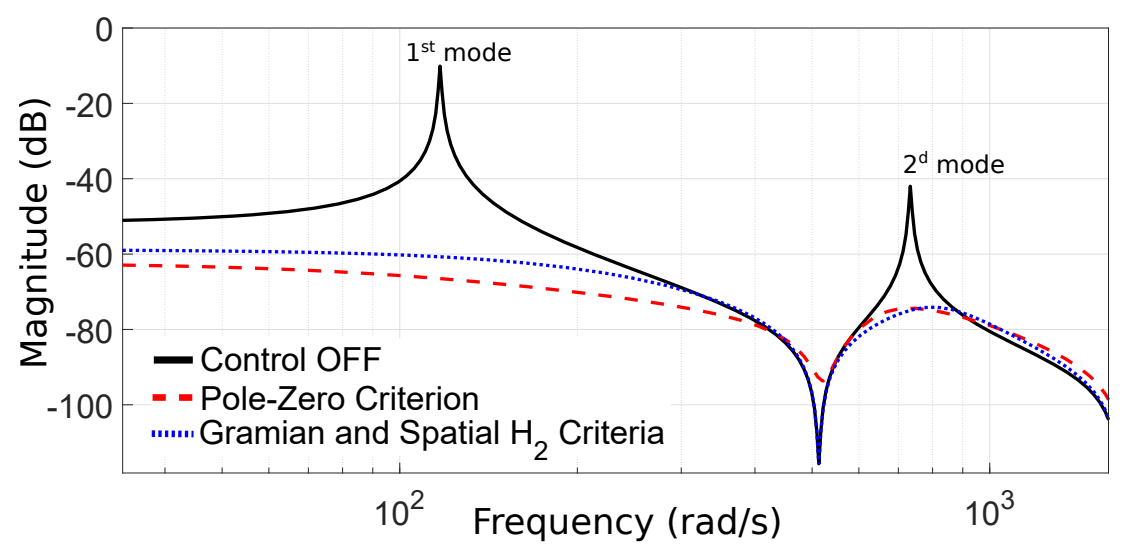

(a) Cantilever Beam

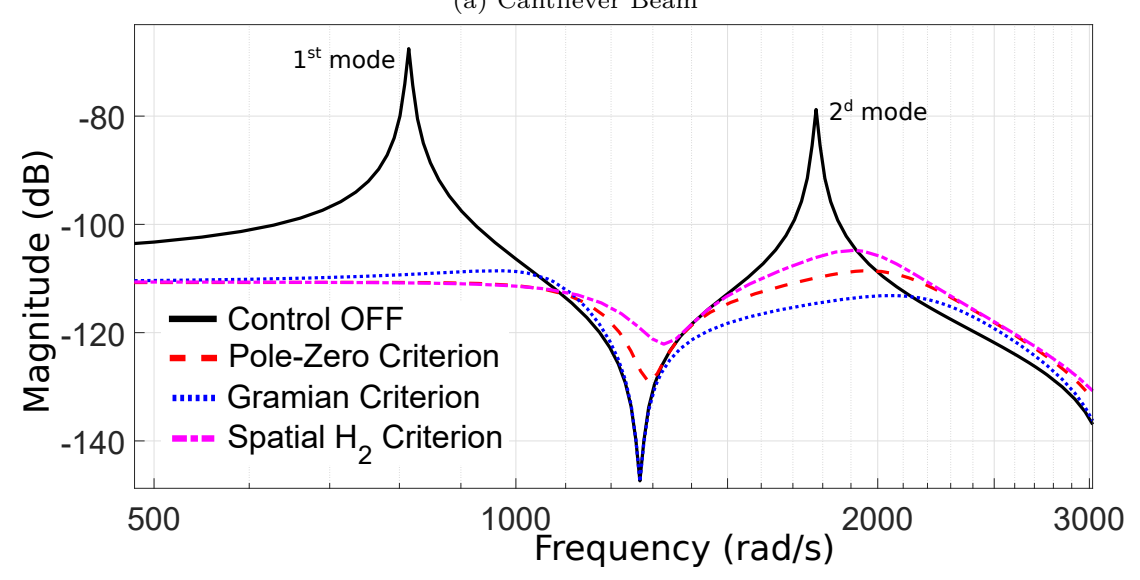

(b) Simply Supported Plate

Figure 11: Frequency Response Functions for Simultaneous Damping of Modes 1 \& 2: (a) Results for the Cantilever Beam, (b) Results for the Simply Supported Plate

The proposed criterion is computed using the zero approximation and its effectiveness is illustrated via numerical simulations for a collocated force actuator/displacement sensor pair on a cantilever beam and on a simply supported plate. The results validate the concept, especially for the cantilever beam for which it is demonstrated that it provides better damping ratios compared to two other popularly used open-loop criteria because it includes information on the modal interactions. For system with higher modal density such as the simply supported plate, improvement on the definition of the criterion should be introduced to better predict singular positions that induce unwanted root locus reshaping. A detailed discussion about this improvement as well as the extension of the proposed criterion to optimal placement of piezoelectric patches would be investigated in future studies.

\section{Acknowledgement}

This research has been funded by the ARC consolidator research program (project: IMpaCT, Integrated Mechatronics for Active vibration ConTrol), Université Libre de Bruxelles, Brussels, Belgium. 


\section{References}

[1] C. Peng, X. Zhou, T. Wei, Y. Ren, High precision synchronous vibration suppression for a msfw subject to phase lag influence, Mechanical Systems and Signal Processing 120 (2019) 408-421. doi:10.1016/j.ymssp.2018.10.017.

[2] C. Liua, X. Jing, S. Daley, F. Li, Recent advances in micro-vibration isolation, Mechanical Systems and Signal Processing 56-57 (2015) 55-80. doi:10.1016/j.ymssp.2014.10.007.

[3] G. Alencar, A. De Jesus, J. G. Santos da Silva, R. Calçada, Fatigue cracking of welded railway bridges: A review, Engineering Failure Analysis 104 (2019) 154-176. doi:10.1016/ j.engfailanal.2019.05.037.

[4] E. A. Al-Bahkali, H. Elkenani, M. Souli, Failure and fatigue life estimate of a pre-stressed aircraft seat support tube, Engineering Failure Analysis 54 (2015) 120-130. doi:10.1016/j. engfailanal.2015.04.001.

[5] K. C. Panda, Dealing with noise and vibration in automotive industry, Procedia Engineering 144 (2016) 1167-1174. doi:10.1016/j.proeng.2016.05.092.

[6] J. Daniel, Automotive wheel and tyre design for suppression of acoustic cavity noise through the incorporation of passive resonators, Journal of Sound and Vibration 467 (2020) 15037. doi:10.1016/j.jsv.2019.115037.

[7] V. Gupta, M. Sharma, N. Thakur, Optimization criteria for optimal placement of piezoelectric sensors and actuators on a smart structure: A technical review, Journal of Intelligent Material Systems and Structures 21 (12) (2010) 1147-1156. doi:10.1177/1045389X10381659.

[8] P. Maghami, S. Joshi, Sensor/actuator placement for flexible space structures, in: American Control Conference, Vol. 2, 1990, pp. 1941-1948.

[9] T. Williams, Transmission-zero bounds for large space structures, with applications, Journal of Guidance, Control, and Dynamics 12 (1) (1989) 33-38. doi:10.2514/3.20365

[10] A. Hac, L. Liu, Sensor and actuator location in motion control of flexible structures, Journal of Sound and Vibration 167 (2) (1992) 239-261. doi:10.1006/jsvi.1993.1333.

[11] I. Bruant, L. Proslier, Optimal location of actuators and sensors in active vibration control, Journal of Intelligent Material Systems and Structures 16 (3) (2005) 197-206. doi:10.1177/ $1045389 \times 05047989$

[12] A. Sadri, J. Wright, R. Wynne, Modelling and optimal placement of piezoelectric actuators in isotropic plates using genetic algorithms, Smart Materials and Structures 8 (1999) 490-498. doi:10.1088/0964-1726/8/4/306 
[13] F. Peng, A. Ng, Y.-R. Hu, Actuator placement optimization and adaptive vibration control of plate smart structures, Journal of Intelligent Material Systems and Structures 16 (3) (2005) 263-271. doi:10.1177/1045389X05050105

[14] K. Lim, Method for optimal actuator and sensor placement for large flexible structures, Journal of Guidance, Control, and Dynamics 15 (1) (1992) 49-57. doi:10.2514/3.20800

[15] W. Gawronski, Advanced Structural Dynamics and Active Control of Structures, Mechanical Engineering Series, Springer-Verlag New York, 2004. doi:10.1007/978-0-387-72133-0.

[16] S. Moheimani, T. Ryall, Considerations on placement of piezoceramic actuators that are used in structural vibration control, in: 38th IEEE Conference on Decision and Control, Vol. 2, 1999, pp. 1118-1123. doi:10.1109/CDC.1999.830077.

[17] D. Halim, S. Moheimani, An optimization approach to optimal placement of collocated piezoelectric actuators and sensors on a thin plate, Mechatronics 13 (1) (2003) 27-47. doi: 10.1016/S0957-4158(01)00079-4.

[18] W. Liu, Z. Hou, M. Demetriou, A computational scheme for the optimal sensor/actuator placement of flexible structures using spatial $h_{2}$ measures, Mechanical Systems and Signal Processing 20 (4) (2006) 881-895. doi:10.1016/j.ymssp.2005.08.030.

[19] S. Devasia, T. Meressi, B. Paden, E. Bayo, Piezoelectric actuator design for vibration suppression - placement and sizing, Journal of Guidance, Control, and Dynamics 16 (5) (1993) 859-864. doi:10.2514/3.21093.

[20] Y. Kang, H. Park, W. Hwang, K. Han, Optimum placement of piezoelectric sensor/actuator for vibration control of laminated beams, AIAA Journal 34 (9) (1996) 1921-1926. doi: $10.2514 / 3.13326$

[21] K. Bendine, F. Boukhoulda, M. Haddag, B. andNouari, Active vibration control of composite plate with optimal placement of piezoelectric patches, Mechanics of Advanced Materials and Structures 26 (4) (2017) 341-349. doi:10.1080/15376494.2017.1387324.

[22] A. Daraji, J. Hale, Reduction of structural weight, costs and complexity of a control system in the active vibration reduction of flexible structures, Smart Materials and Structures 23 (9) (2014) 095013. doi:10.1088/0964-1726/23/9/095013

[23] K. R. Kumar, S. Narayanan, The optimal location of piezoelectric actuators and sensors for vibration control of plates, Smart Materials and Structures 16 (6) (2007) 2680-2691. doi: $10.1088 / 0964-1726 / 16 / 6 / 073$.

[24] G. D. Martin, On the control of flexible mechanical systems, Ph.D. thesis, Stanford University (1978).

[25] A. Preumont, Vibration Control of Active Structure, An Introduction, thrid edition Edition, Springer, 2011. doi:10.1007/978-94-007-2033-6 
[26] D. Miu, Physical interpretation of transfer function zeros for simple control systems with mechanical flexibilities, Journal of Dynamic Systems, Measurement, and Control 113 (3) (1991) 419-424. doi:10.1115/1.2896426.

[27] T. Williams, Constrained modes in control theory: Transmission zeros of uniform beams, Journal of Sound and Vibration 156 (1) (1992) 170-177. doi:10.1016/0022-460X(92)90819-J.

[28] W. Wolovich, On determining the zeros of state-space systems, IEEE Transactions on Automatic Control 18 (5) (1973) 542-544. doi:10.1109/TAC.1973.1100379

[29] E. Davison, S. Wang, Properties and calculation of transmission zeros of linear multivariable systems, Automatica 10 (6) (1974) 643-658. doi:10.1016/0005-1098(74)90085-5.

[30] A. Emami-Naeini, P. Van Dooren, Computation of zeros of linear multivariable systems, Automatica 18 (4) (1982) 415-430. doi:10.1016/0005-1098(82)90070-X.

[31] F. M. Fleming, The effect of structure, actuator, and sensor on the zeroes of controlled structures, Ph.D. thesis, Massachusetts Institute of Technology (1991).

[32] E. Balmes, Structural dynamics toolbox 7.1 (for use with matlab) (1991).

[33] B. Boashash, Time-Frequency Signal Analysis and Processing, A Comprehensive Reference, second edition Edition, Academic Press, 2016.

[34] W. Levine, The Control Handbook in two Volumes, CRC Press, 1999. 